\title{
电刺激中央灰质区、尾核和穴位电针对 清醒家兔丘脑后核群诱发放电的抑制
}

\author{
赵志奇 郡殿华* 杨振荃 \\ (中国科学院上海脑研究所)
}

急性实验资料证明丘脑后核群 (PO) 神经元参与伤害性信息的传递 ${ }^{[1,2]}$. 本文报道在慢 性实验中 PO 神经元的伤害性放电和动物伤害性行为反应的相互关系及脑刺激和电针的抑制 效应.

实验动物为 12 只成年家兔. 在麻醉下按照 Sawyer 图谱埋植脑刺激电极和 MWT-1 型慢 性微电极推进器的基座 ${ }^{[3]}$. 手术后三天开始实验观察. 观察时动物放在木制的兔箱内, 头和 身体可在一定范围内活动. 每次实验前将微推进器安装在基座上. 用玻璃绝缘的铇丝电极 (尖端直径 15 微米左右) 记录 PO 区的多单位放电, 少数实验用玻璃电极 (1-2 微米) 记录单 位放电. 在动物尾巴两侧的皮下插人一对尖端裸露的绝缘不锈钢毫针, 通以一串方波 (串长 10-30 毫秒, 频率 100-300 赫, 电压 20-25 伏), 引起动物明显的全身反应. 如: 屈腿, 抖动 身体,甩耳,摇头,甚至全身挣扎等. 简称为“伤害性行为反应”。

单位放电经过固定在微推进器基座上的一个高阻抗低噪声的场效应管, 再输入到放大器, 以减小运动伪迹. 实验结果记录在调频的记录仪上. 处理资料时, 将录制的 PO 区的多单位 放电先输至“生物电脉冲限幅器”[4], 选取欲处理的脉冲, 去除电针与脑刺激的伪迹, 再输人到 电子计算机进行分析. 实验结束时, 采用常规的电介损毁法作刺激电极和记录电极的尖端定 位, 玻璃电极尖端则用微电泳注射 HRP 定位.

丘脑后核群 $(P O)$ 的单位反应 在内膝体的巨细胞群 $(M G m)$ 和膝上核 $(S G)$ (定位坐标 为: $P$ 6, $\left.L_{\mathrm{L}} 5, \mathrm{H}-3\right)$ 记录神经元的发放. 在动物清醒安静的状态下, $P O$ 神经元呈节律性自 发放电, 而当动物䀩盹时, 往往出现阵发性成簇的放电. 在 PO 中, 对刺激有反应的单位大约 不超过三分之一. 和急性实验结果相似,多数单位具有异觉会聚和异位会聚的特点,对于轻机 械刺激, 弱电流刺激和伤害性刺激以及声音均有反应. 反应的感受野很大, 呈双侧性, 缺乏明 显的区域定位. 对伤害性刺激反应的单位, 常伴随有动物伤害性行为的变化, 表现为放电频率 显著增加, 呈现长串反应 (持续数百裹秒以上). 这类神经元的反应特点与脊髓的广动力范围 的单位反应颇为相似 ${ }^{[5]}$ 、往往与伤害性传入活动有关 ${ }^{[1]}$. 给七只动物静脉注射镇痛剂芬太尼 (30 微克/公斤体重), 伤害性行为反应均受明显抑制, 记录的 11 个长串反应的神经元中 8 个 被抑制. 这类神经元还可以为动脉注射致痛物质徐缓激肽所激活 ${ }^{[3]}$. 但动物在自发运动或非 伤害性被动运动时, 不出现长串反应. 我们相信这类神经元的活动与伤害性信息传递有关.

对串刺激诱发的 42 个 PO 神经元的反应, 结合动物伤害性行为反应的变化, 观察了脑刺

本文 1980 年 8 月 26日收到.

*中国科学院上海生理研究所. 
激和穴位电针的影响. 实验步骤:（1）在刺激前, 先记录动物安静状态和自发运动时 PO 神经 元放电作为背景活动; (2) 每隔 20 秒给动物一次伤害性刺激, 以连续三次诱发的行为和 PO 放电为对照反应; (3)记录脑刺激和电针期间及停止刺激以后上述反应的变化.

脑刺激的抑制效应脑刺激用恒流重复方波刺激(波宽 0.1 毫秒,频率 5-50 赫).中央 灰质 (PO、LO、 $\mathrm{H}-1)$ 和尾核头部 $\left(A 5 、 L_{R} 3 、 H+4\right)$ 的刺激强度分别为 100-600 微安 和 $200-1000$ 微安.

（i）电刺激中脑中央灰质区 (CG): 已知脑干内侧网状结构在痛觉调制中有重要作用 ${ }^{[6]}$. 我们在 9 只动物上比较了刺激 CG 前后, 串刺激诱发的 PO 神经元放电和伤害性行为反应的变 化. 开始刺激 CG 时动物往往出现睁眼、坚耳朵等似乎“警觉”的反应, 数十秒后, 动物较安 静, 此时对伤害性行为反应有强抑制, 串刺激尾巴只能引起动物轻微的抖动, 有的动物甚至对 伤害性刺激毫无反应, 与对照反应有非常明显的差异. 这种抑制现象往往在脑刺激停止后的 1-2 分钟内更甚, 并有一个从几分钟到几十分钟的抑制后效应. 伴随上述变化, 串刺激诱发 的 29 个单位中 19 个单位的放电和行为反应有大体平行的抑制发展和恢复过程. 图 1 是其中 一个单位的单位发放的密度序列直方图, 在脑刺激期间 PO 放电明显抑制(图 $1 \mathrm{~B}$ ), 停止刺激 后有后效应, 反应逐渐恢复. 有少数单位, 串刺激引起动物伤害性行为反应时, 自发放电抑制, 刺激 CG 伴随行为反应的抑制, 自发放电的抑制反而解除.

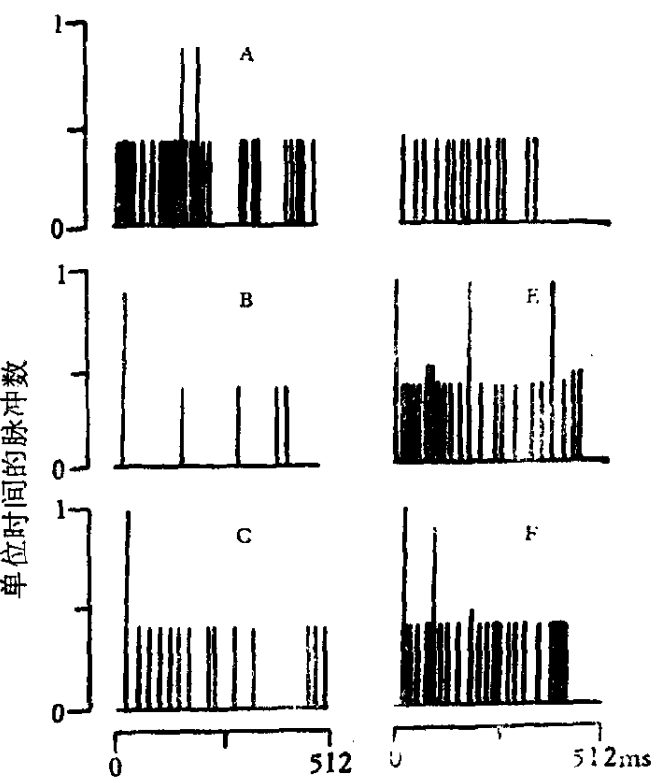

图 1 电刺激中央灰质 (CG) 对虫刺激尾巴诱发的 PO 放电的抑制,

由三次记录叠加描制的密度序列直方图

横座标为时间 512 毫秒, 纵座标为单位时间 (2 毫秒)的脉冲数. A 对炤反应, B 电 刺激中央灰质时, C、D、E 和 F 分别为停止刺激后 $1 、 5 、 10$ 和 20 分钟时

（ii）尾核头部 (Cd) 是参与镇痛的一个重要结构 ${ }^{[7]}$. 我们在 3 只动物上观察了 9 个细胞 的反应, 刺激尾核头部时, 伴随串刺激诱发的动物伤害性行为反应的强抑制, 7 个 PO 神经元 的长串反应亦明显减少. 图 $2 \mathrm{I}$ 表明刺激尾核头部时一个 PO 神经元放电的变化. 这个单位 也为静脉注射芬太尼所抑制 (图 2 II).

电针的抑制效应电针双侧“环跳”穴, 频率 5-8 赫, 强度 1-5 毫安. 实验观察到电针 

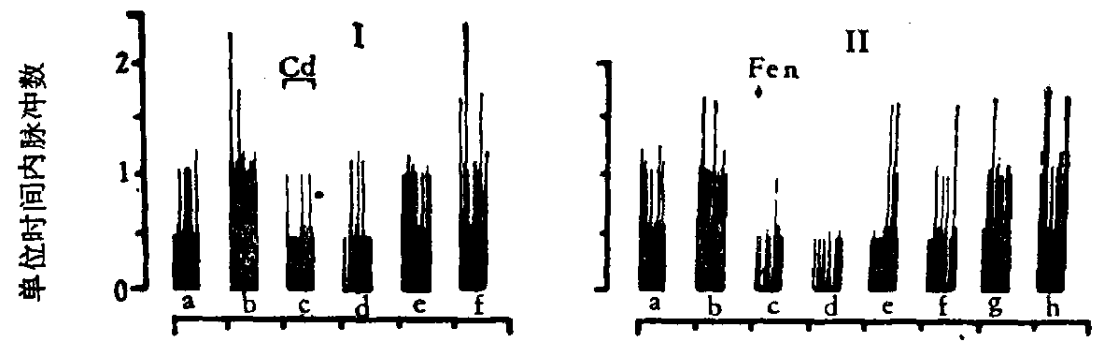

时间：每小格 1024 毫秒

图 2 电刺激尾核头部 (CD) 和静脉注射芬太尼对 PO 伤害性放电的抑制

I、II 分别为刺激 CD 和注射芬太尼 (30 微克/公斤) 时 PO 放电的密度序列直 方图(三次叠加). 单位时间 2 毫秒,分析时间 512 毫秒.

I: $\mathrm{a}$ 自发放电, $\mathrm{b}$ 对照反应, $\mathrm{c}$ 电刺激尾核时, $\mathrm{d} 、 \mathrm{e}$ 和 $\mathrm{f}$ 分别为停止刺激后 1 、 5 和 10 分钟;

II: $a$ 自发放电, $b$ 对琞反应， $c 、 d 、 e 、 f 、 g$ 和 $h$ 分别为注射后 $2 、 5 、 10 、 20 、 40$ 和 50 分钟

对动物伤害性行为反应的抑制一般较弱. 和脑刺激的效应不同.电针的抑制有一个发展过程, 需要 20-30 分钟的诱导期, 此时, 动物表现为对伤害性刺激的反应减弱, 停止电针后有几十分 钟的后效应, 在刚停电针的头几分钟内动物尤为安静. 在上述过程中 PO 神经元的诱发放电 也有相应的变化. 图 3 是一次实验中 PO 神经元放电的密度序列直方图, 电针诱导后诱发放 电减少,停电针后 50 分钟恢复到对照水平。在恢复过程中, 动物往往出现 “目园盹” 状态, 闭眼 垂头、很少有自发运动. $\mathrm{PO}$ 神经元出现阵发性成簇的放电. 串刺激诱发的放电反应型式亦有 所改变.

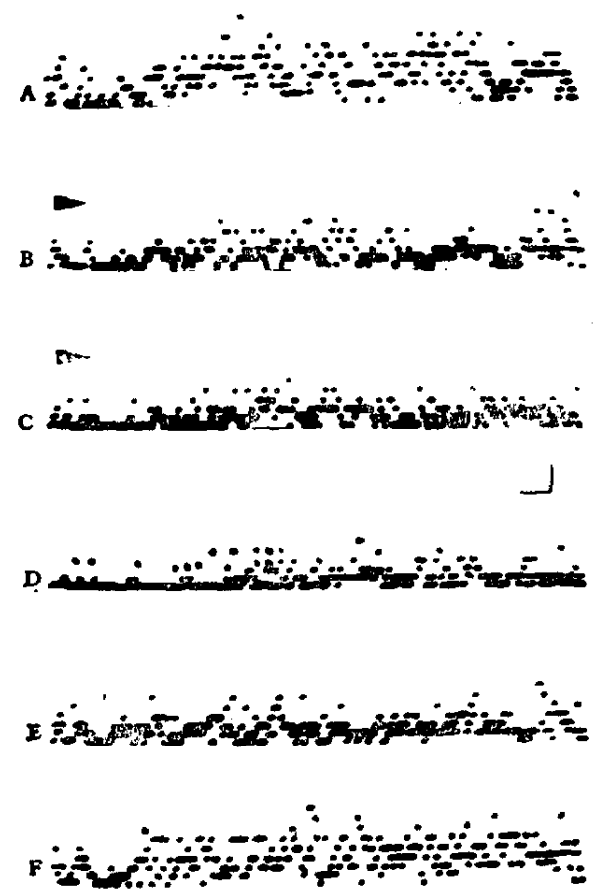

图 3 穴位电针时一个 PO 神经元伤害性发放的密度序列直方图（三次反应叠加） $A$ 对照反应, $b 、 c$ 为电针双侧“环跳”穴 20 和 30 分钟时, $d 、 e$ 和 $f$ 为停电针后 $5 、 30$ 和 50 分钟时. 校正: 横座标为时间 77 旁秒,纵坐标为单位时间(5 毫秒)内的脉冲数 (1 个脉冲) 
在脑刺激或电针的作用下, 动物伤害性行为反应受抑制时, 大多数的 PO 神经元诱发放电 亦相应地抑制. 中央灰质刺激所需的电流最小, 而抑制作用最强, 尾核次之, 电针作用相对较 弱, 而且需要一个较长的诱导期. 这些结果表明, PO 神经元参与伤害性信息的传递, 其放电 的抑制一定程度上反映了疼痛的变化.

脑刺激和电针通过什么途径影响 PO 神经元的反应呢? 已知道, 静脉注射或中央灰质内 微量注入纳络酮可部分阻断刺激尾核 ${ }^{[8]}$, 中央灰质 ${ }^{[0]}$ 和电针 ${ }^{[10]}$ 产生的镇痛作用, 说明可能是通 过内腓肽释放的机制. 我们在中央灰质和尾核的刺激部位可记录到电针传入冲动诱发的场电 位, 提示电针信号可到达上述结构. 因此, 有理由设想, 中央灰质, 尾核和电针对 PO 神经元发 放的抑制, 至少部分是由于激活了内啡肽系统的活动, 经下行抑制通路阻断了脊髓背角伤害性 信息向 PO 神经元的传递 ${ }^{[11,12]}$. 此外, 在实验中还观察到刺激中央灰质和尾核时, 在 PO 记录 到诱发的场电位. 这就提示了另一可能性, 即 PO 神经元的抑制, 是由于来自中央灰质和尾核 的传人信号, 在丘脑后核群内与伤害性信号发生相互作用的结果. 在急性实验中 ${ }^{[13]}$, 切断脊䯈 的下行抑制通路后，电针和局部脑刺激对束旁核痛放电的抑制并不消失. 这一事实也利于 上述的推论.

饶人兴味地是中央灰质和尾核刺激对 PO 神经元的声、光诱发放电也有明显的抑制作用. 这似乎说明, 尽管中央灰质有大量吗啡受体和内啡肽的存在, 使其在痛觉调制中占有特殊地 位, 但是, 像这样一个有着广泛传入传出解剖联系的神经结构, 在外源性电流刺激下, 不可能只 专一性地影响痛觉的传递, 只是在一定条件下,可能对痛觉的抑制更明显.

\section{参考文 献}

[1] Peggio, G. F. et al., Johns. Hopk. Hosp. Bull., 106(1960), 266.

[ 2 ] Guilbaud, G. C. et al., Archiv. Ital. Biol., 115(1977), 38.

[3] 孙公铎等, 科学通报, 23(1978), 7: 447 .

[4] 陈俊强等, 生理学报, 32(1980), 87-91.

[5] 吴建屏等, 中国科学, 1974, 5: 526 .

[6] Mayer, D. J. et al., Pain, 2(1976), 379.

[ 7 ] 上海生理研究所, 中华医学杂志, $1975,5: 34$.

[8] 何莲芳等, 全国针炎针麻学术讨论会论文摘要(1), 北京, 1979,23 .

[9] Akil, H. et al., Science, 191 (1976), 961.

[10] Pomeranz, B, et al., Exp. Neurol, 64(1979), 327.

[11] 沈锷等, 中国科学, 1979, 2: 211.

[12] Carstens, E. et al., J. Neurophsiol, 42(1979), 558.

[13] 张桂林等, 全国针众针麻学术讨论会论文摘要(2), 北京, 1979, 54 . 